\title{
Algebraic decay of velocity fluctuations near a wall
}

\author{
I. Pagonabarraga, ${ }^{1}$ M. H. J. Hagen, ${ }^{1, *}$ C. P. Lowe, ${ }^{1,2}$ and D. Frenkel ${ }^{1}$ \\ ${ }^{1}$ FOM Institute for Atomic and Molecular Physics, Kruislaan 407, 1098 SJ Amsterdam, The Netherlands \\ ${ }^{2}$ Computational Physics, Delft University of Technology, Lorentzweg 1, 2628 CJ Delft, The Netherlands
}

(Received 23 June 1998)

\begin{abstract}
Computer simulations of the dynamics of a colloidal particle suspended in a fluid confined by an interface show that the asymptotic decay of the velocity correlation functions is algebraic. The exponents of the longtime tails depend on the direction of motion of the particle relative to the surface, as well as on the specific nature of the boundary conditions. In particular, we find that for the angular velocity correlation function, the decay in the presence of a slip surface is faster than the one corresponding to a stick one. An intuitive picture is introduced to explain the various long-time tails, and the simulations are compared with theoretical expressions where available. [S1063-651X(98)00612-6]
\end{abstract}

PACS number(s): 66.20. + d, 83.20.Jp, 05.40.+j, 82.70.Dd

\section{INTRODUCTION}

The dynamical behavior of bulk colloidal suspensions is, on the whole, fairly well understood [1]. By means of diffusing wave spectroscopy (DWS) $[2,3]$, the time regime in which hydrodynamic modes relax has been studied, and even in this regime, experiments and theory are in agreement. Whereas DWS has been applied to study the dynamics in bulk suspension, the effect of confining surfaces on the dynamics is less well understood, despite its relevance in situations of great practical interest, such as diffusion in porous media.

In this paper we concentrate on the simplest possible confining geometry, namely, a semi-infinite fluid confined by a planar interface. It is well known that the presence of a single wall modifies the hydrodynamic response of the fluid, and it has been suggested that this feature is used by some microorganisms to enhance their motility [4]. We will focus on the dynamics of colloidal particles close to a surface at time scales short compared to the time it takes the particles to displace over a distance comparable to their own radius. This corresponds to the "short-time" regime in the dynamics of colloidal suspensions, in which hydrodynamic excitations decay. In this regime, the particles experience the influence of such modes. When, in what follows, we refer to "long times," we mean the time scale of the asymptotic decay of this "short-time" regime. For a typical suspension, these times are of order $\sim 10^{-8} \mathrm{~s}$, while the time it takes a particle to displace over a distance equal to its radius is much longer $\left(\sim 10^{-3} \mathrm{~s}\right)$.

Until the early 1970 s it was thought that the decay of the velocity of a suspended particle would be exponential. However, in their pioneering work, Alder and Wainwright [5] showed that the velocity autocorrelation function (VACF), $C_{v}(t)=\langle\vec{v}(t) \cdot \vec{v}(0)\rangle$, of a tagged particle in a hard-sphere fluid moving with velocity $\vec{v}(t)$ exhibited an algebraic decay at long times, $C_{v}(t)=(d-1) M C_{v}(0) /[d \rho(4 \pi(\nu$

\footnotetext{
*Present address: Unilever Research, Port Sunlight Laboratory, Quarry Road East, Bebington, Wirral L63 3JW, U.K.
}

$\left.+D))^{d / 2} t^{d / 2}\right]$, where $d$ is the dimensionality of the system, $M$ the mass of the particle, $\rho$ and $\nu$ are the density and the kinematic viscosity of the solvent, respectively, and $D$ is the diffusion coefficient of the tagged particle. As was already argued by Alder and Wainwright, this behavior is quite general as a consequence of the conservation of momentum. The argument also implies that the angular velocity autocorrelation function (AVACF) of a tagged particle with angular velocity $\omega(t), \quad C_{\omega}(t)=\langle\omega(t) \cdot \omega(0)\rangle$, will decay algebraically at long times, $C_{w}(t) \sim 1 / t^{d / 2+1}[6]$, and this has indeed been found in computer simulations [7]. The study of correlation functions is also interesting because their integrals are related to transport coefficients, connecting thus the dynamical properties of the constituent particles of the system with its macroscopic response. In particular, the time-dependent diffusion coefficient is obtained as the integral of the VACF.

The effect that a bounding surface has on the decay of the velocity of an initially moving particle has been analyzed theoretically by Gotoh and Kaneda [8]. These authors considered the VACF of a single sphere of radius $r$ at a distance $h$ from a stick surface, i.e., an interface where the velocity of the fluid and the surface are equal. They showed, by a perturbative calculation that becomes exact for asymptotically long times, that the wall generates both an anisotropic relaxation and that it modifies the power law of the long-time algebraic decay. If the time needed for vorticity to diffuse the distance between the particle and the wall is $\tau_{w} \equiv h^{2} / \nu$, they predict for times $t \gg \tau_{w}$,

$$
C_{v}^{\|}(t)=\frac{A_{\|}}{t^{5 / 2}}+O\left(\frac{1}{t^{7 / 2}}\right), \quad C_{v}^{\perp}(t)=\frac{A_{\perp}}{t^{7 / 2}}+O\left(\frac{1}{t^{9 / 2}}\right)
$$

with the amplitudes given by

$$
A_{\|}=\frac{M C_{v}^{\|}(0)}{\nu \rho} \frac{h^{2}}{(4 \pi \nu)^{3 / 2}}, \quad A_{\perp}=\frac{M C_{v}^{\perp}(0)}{4 \nu^{2} \rho} \frac{h^{4}}{(4 \pi \nu)^{3 / 2}},
$$

where $C_{v}^{\|}(0)$ and $C_{v}^{\perp}$ are the initial values of the VACF for a particle moving initially parallel and perpendicular to the wall, respectively. 
One may expect that the change in boundary conditions at the surface will make a qualitative difference for the dynamics of the colloids. For example, one may consider a slip interface, where the tangential component of the stress is continuous across the surface, and which is characteristic of a fluid/fluid interface. In fact, there exist examples where the dynamics of colloidal particles is sensitive not only to the confinement, but to the specific behavior of the velocity field at the interface [9].

Therefore, we have performed computer simulations of the short-time dynamics of a particle in the presence of both a slip and a stick surface. We have focused both on the VACF and AVACF, as these two functions are most directly linked to experimentally observable quantities. Before presenting our results in Sec. III, we first introduce an intuitive picture that will allow us to interpret our results, and provides a physical way of understanding the modifications that a bounding surface induces in the relaxation of the hydrodynamic modes. We conclude the paper with a discussion of our results.

\section{THEORETICAL DESCRIPTION}

The short-time dynamics of a colloidal particle is controlled by the decay of the longest-lived hydrodynamic mode in the solvent. In the case of an unbounded fluid, the local amplitude of the sound modes excited initially by the particle decays much faster than the vorticity, which relaxes diffusively. The algebraic tail $C_{v}(t) \sim 1 / t^{d / 2}$ can be simply understood as a coupling of the velocity of the particle to the diffusion of vorticity [10]. The velocity field $\vec{v}$ of a low Reynolds number incompressible flow satisfies the diffusion equation,

$$
\frac{\partial \vec{v}(\vec{r}, t)}{\partial t}=\nu \nabla^{2} \vec{v}(\vec{r}, t)
$$

A particle of mass $M$ located originally at $\vec{r}_{0}$ and with velocity $\vec{u}_{0}$, will create a flow field in the fluid. This field is equal to the one generated by a small fluid element $\delta V$ centered in $\vec{r}_{0}$, with initial velocity $\vec{v}_{0}$ such that $M \vec{u}_{0}$ $=\rho \vec{v}_{0} \delta V$. In the limit $\delta V \rightarrow 0$, the initial velocity field is $\vec{v}(\vec{r}, t=0)=\left(M \vec{u}_{0} / \rho\right) \delta\left(\vec{r}-\vec{r}_{0}\right)$. From Eq. (3), it then follows

$$
\begin{aligned}
\vec{v}(\vec{r}, t)= & \left\{\frac{\exp \left[-\frac{r^{2}}{4 \nu t}\right]}{(4 \pi \nu t)^{d / 2}}(\mathbf{1}-\hat{r} \hat{r})\right. \\
& \left.-\frac{1}{2\left(\pi r^{2}\right)^{d / 2}} \gamma\left(\frac{d}{2}, \frac{r^{2}}{4 \nu t}\right)(\mathbf{1}-d \hat{r} \hat{r})\right\} \cdot \vec{v}_{0},
\end{aligned}
$$

where $\gamma$ is the incomplete gamma function. From the previous expression, it is easy to check that indeed the velocity induced by a localized perturbation decays asymptotically as $1 / t^{d / 2}$. The presence of a single bounding surface will not disturb the diffusive decay of the velocity at long times due to vorticity. The only feature we have to take into account is the fact that the wall will modify the induced flow field. We

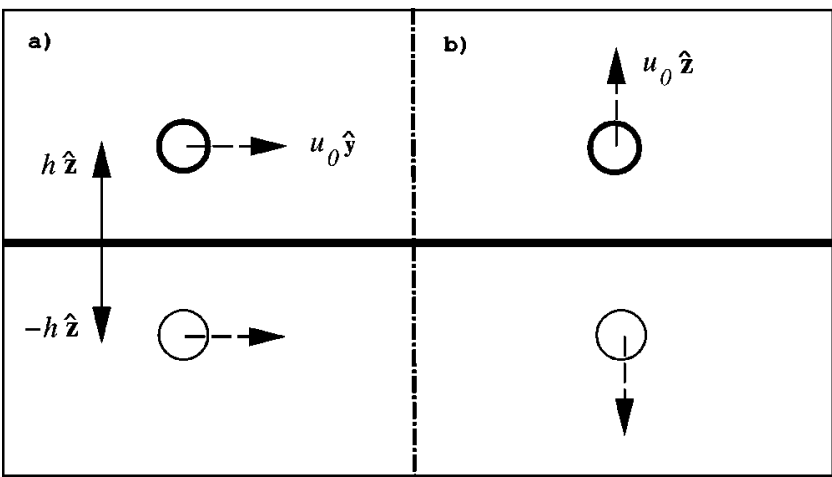

FIG. 1. Image particle for a translating colloid above a slip surface, placed initially at $h \hat{z}$. (a) Parallel motion, (b) perpendicular motion.

account for this by using the standard method of images. In this method, one places additional sources in the region of space not accessible to the system to enforce the desired boundary condition at the interface [11]. However, a simple point image, as is used when considering mass diffusion or electrostatic potentials, is not sufficient to enforce a boundary condition for a vectorial field, such as the velocity. To be more precise, for slip boundary conditions, we need only impose the condition that there is no flux of momentum across the interface. This can be accomplished by a simple image particle moving with the appropriate velocity. For stick boundary conditions, however, all the components of the velocity have to vanish at the surface. In this case, a simple point image is not enough to fulfill the conditions at the wall. Blake [4] worked out the complete set of images needed to reproduce the steady flow corresponding to an initial point source. It has even been argued that at finite frequency, the flow in the presence of a solid wall cannot be described with a finite set of image singularities [12]. Nonetheless, rather than attempting a rigorous description of vorticity diffusion in the presence of a solid surface, in this simplified theory we will consider the simplest image set necessary to provide the basic features of the long-time hydrodynamic behavior. Therefore, we will characterize the solid surface by imposing that no momentum can be transported along it. This description produces a boundary condition that resembles more a porous surface rather than a real solid wall, because there can still be momentum flux across the wall, but it contains the basic feature that a solid surface resists the flow of fluid along it. The comparison with the simulations will show that this assumption predicts the proper temporal decay at long times, although it is in general quantitatively inaccurate, as could be expected.

Let us first consider that the particle is placed at a distance $h \hat{z}$ above a slip interface located at $z=0$. If at time $t=0$ the particle has a velocity $u_{0} \hat{y}$, at later times the velocity at the same point will be expressed as the sum of the velocity generated by the particle itself plus the one generated by an image source located at $-h \hat{z}$ moving with the same velocity $u_{0} \hat{y}$, as displayed in Fig. 1(a). As can be derived from Eq. (4), the long-time decay is given by

$$
u_{\|}^{\mathrm{sl}}(t)=\frac{2(d-1)}{d} \frac{v_{0}}{(4 \pi v t)^{d / 2}}+O\left(\frac{1}{t^{d / 2+1}}\right) .
$$


In order to avoid confusion when we consider later a stick surface, we have used the superscript "sl" to indicate that the particle is moving in the presence of a slip interface, as opposed to the superscript "st" labeling a stick surface.

The interface does not modify the power of the temporal decay in this particular situation. However, if the particle moves perpendicularly to the interface, then the image particle has to move in the opposite direction, as shown in Fig. 1(b) In this case, the velocity at long times is given by

$$
u_{\perp}^{\mathrm{sl}}(t)=\frac{4 \pi v_{0} h^{2}}{(d+2)(4 \pi \nu t)^{d / 2+1}}+O\left(\frac{1}{t^{d / 2+2}}\right),
$$

which shows that the interface induces a faster decay of the $\mathrm{VACF}$, due to the interaction with the image.

If we consider a wall where stick boundary conditions are satisfied, then the images should be modified accordingly. If the particle is moving initially at a distance $h \hat{z}$ and parallel to the wall with velocity $u_{0} \hat{y}$, the image, located at $-h \hat{z}$, has to move in the opposite direction with velocity $-u_{0} \hat{y}$. We then get asymptotically,

$$
u_{\|}^{\mathrm{st}}(t)=\frac{6 \pi}{(d-1)(d+2)} \frac{v_{0} h^{2}}{(4 \pi \nu t)^{d / 2+1}}+O\left(\frac{1}{t^{d / 2+2}}\right),
$$

which for three dimensions (3D) coincides with the decay predicted by Eq. (1), and recovers the proper dimensional behavior of the amplitude. However, as already mentioned, with this intuitive approach the numerical factor of the tail does not coincide with the prediction of Eq. (2).

For motion perpendicular to the interface, we need to take a quadrupole in order to impose that there is no flux parallel to the wall. For example, in three dimensions, besides the image at $-h \hat{z}$ with velocity $-u_{0} \hat{z}$, one can enforce a vanishing parallel velocity at the wall by placing an image ring on the surface at a distance $2 h$ from the origin, with a strength twice the strength of the initial source, pointing away from the origin. In this case, the combination of the sources gives

$$
u_{\perp}^{\mathrm{st}}(t)=A \frac{v_{0} h^{4}}{(4 \pi \nu t)^{d / 2+2}}+O\left(\frac{1}{t^{d / 2+3}}\right)
$$

where $A=24 \pi^{2} / 7$ in $3 \mathrm{D}$. It is worth noting that the algebraic decay is characterized by a large power, 7/2 in three dimensions. Clearly, the stick wall always induces a faster decay of the velocity than the slip interface for motion in any direction, since it damps momentum more efficiently than a liquid interface. For both kinds of interfaces, the motion perpendicular to the surface always decays faster.

We can also use this intuitive approach to analyze how the interface will modify the decay of the angular velocity autocorrelation functions. In this case, we should look at the decay of the angular velocity if initially a torque is applied at the particle. If we call $\hat{n}$ a vector normal to the surface of the particle, located at $\vec{r}_{0}$, the angular velocity $\vec{\omega}$ induced by an initially applied force distribution, $\vec{F}(\vec{r}, t)=\vec{F}\left[\delta\left(\vec{r}-\vec{r}_{0}-\vec{n}\right)\right.$ $\left.-\delta\left(\vec{r}-\vec{r}_{0}+\vec{n}\right)\right] \delta(t)$, can be shown to satisfy [13]

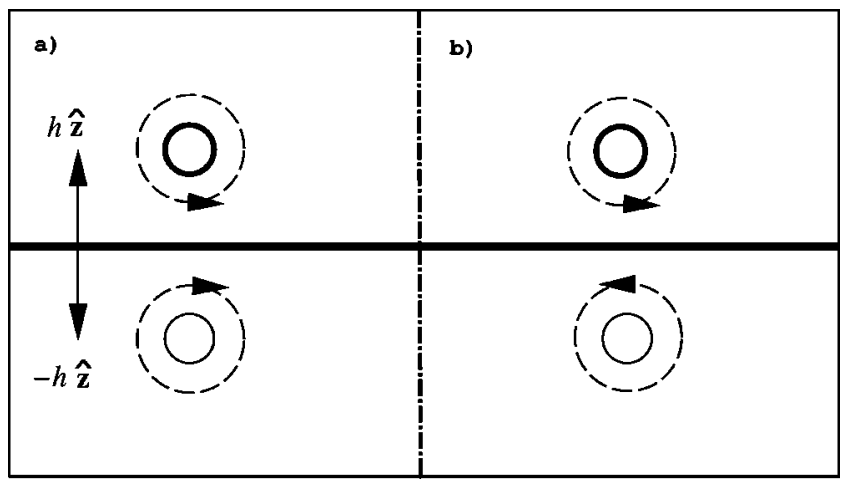

FIG. 2. Image set for a disk above a surface rotating with its axis parallel to the wall, placed initially at $h \hat{z}$. (a) Slip surface, (b) stick surface.

$$
\vec{\omega} \times \vec{r}=\int e^{-\nu k^{2} t} e^{i \vec{k} \cdot\left(\vec{r}-\vec{r}_{0}\right)}(\mathbf{1}-\hat{k} \hat{k}) \cdot\left(I \vec{\Omega}_{0}\right) \sin (\vec{k} \cdot \vec{n}) d \vec{k}
$$

where the vector $\vec{r}$ means a vector joining the center of the object to a point on its surface. Moreover, $I$ is the moment of inertia of the particle and $\vec{\Omega}_{0}$ is the initial angular velocity of the fluid, which is related to the total initial torque $\vec{T}$ applied by the force distribution $\vec{F}$ through $\vec{T}=\int d \hat{n} \vec{F} \times \vec{n}$ $\equiv I \vec{\Omega}_{0} \delta(t)$. Note that there is no net momentum transmitted to the system. Similar to the case of the velocity, the magnitude of the torque is related to the initial angular velocity of the particle in such a way that $\vec{\Omega}=\vec{\omega}_{0} / \rho$, where $\vec{\omega}_{0}$ is its initial angular velocity.

Let us first consider that the colloid rotates in the presence of a slip surface. If the axis of rotation is parallel to the interface, then in order to ensure that there is no component of the velocity field through the interface, we should place an image vortex which rotates in the opposite sense, as depicted in Fig. 2(a). According to Eq. (9), this initial perturbation gives rise to an asymptotic decay of the angular velocity

$$
\omega_{\|}^{\mathrm{sl}}(t)=\frac{3(4 \pi)^{2}}{d+1} \frac{h^{2} T}{(4 \pi \nu t)^{d / 2+2}}+O\left(\frac{1}{t^{d / 2+3}}\right) .
$$

If the axis of rotation is perpendicular to the interface, a corotating source and image suffice to ensure that there is no net flux of momentum across the interface. This combination leads to a slower decay of the vorticity than in the previous geometry, namely,

$$
\omega_{\perp}^{\mathrm{sl}}(t)=\frac{8 \pi}{d+1} \frac{T}{(4 \pi \nu t)^{d / 2+1}}+O\left(\frac{1}{t^{d / 2+2}}\right)
$$

In the case of stick boundary conditions, when the axis of rotation is parallel to the interface, the velocity along the wall is zero if we take a corotating image particle, as displayed in Fig. 2(b) In this case the decay is then

$$
\omega_{\|}^{\mathrm{st}}(t)=\frac{8 \pi}{d+1} \frac{T}{(4 \pi \nu t)^{d / 2+1}}+O\left(\frac{1}{t^{d / 2+2}}\right),
$$


TABLE I. Powers of the algebraic tail for the VACF and the AVACF in $d$ dimensions. For $d=2, C_{\omega}^{\perp}$ is not defined.

\begin{tabular}{cccc}
\hline \hline & slip & stick & unbounded \\
\hline$C_{v}^{\| !}$ & $\frac{d}{2}$ & $\frac{d}{2}+1$ & \\
$C_{v}^{\perp}$ & $\frac{d}{2}+1$ & $\frac{d}{2}+2$ & $\frac{d}{2}$ \\
$C_{\omega}^{\| l}$ & $\frac{d}{2}+2$ & $\frac{d}{2}+1$ & \\
$C_{\omega}^{\perp}$ & $\frac{d}{2}+1$ & $\frac{d}{2}+2$ & $\frac{d}{2}+1$ \\
\hline \hline
\end{tabular}

which coincides with Eq. (11), and has an amplitude which is twice the one corresponding to a rotating particle in an unbounded fluid. Finally, if the axis is perpendicular to the interface, the image should rotate in the opposite sense in order to avoid the flow along the wall. This leads then to

$$
\omega_{\perp}^{\mathrm{st}}(t)=\frac{(4 \pi)^{2}}{d+1} \frac{h^{2} T}{(4 \pi \nu t)^{d / 2+2}}+O\left(\frac{1}{t^{d / 2+3}}\right)
$$

In this particular case, due to the symmetry of the two sources, all the components of the velocity will vanish at the solid surface. This implies that the amplitude also has to be asymptotically correct in this case.

The previous analysis shows that the AVACF behaves differently close to an interface than the VACF. For an unbounded fluid, the AVACF always decays with one power higher than the corresponding VACF. A stick surface always produces a faster decay on the VACF than a slip interface. The behavior of the AVACF in the presence of an interface is not simply related to the corresponding VACF. In fact, we arrive at the rather surprising prediction that, for rotation parallel to the interface, slip boundary conditions yield a faster decay of the AVACF than stick boundary conditions.

In Table I we have summarized the different exponents of the VACF and AVACF as a function of the geometry and the boundary conditions.

Thus far we have focused on the decay of the velocity field generated by a moving particle, disregarding its Brownian motion. Diffusion of the colloid during the decay of the flow field will lead to mode coupling between flow and diffusion. For an unbounded fluid, this so-called mode-coupling effect modifies the amplitude of the correlation functions, but not the power law characterizing the long-time tail [14]. Using mode coupling theory, it is easy to show that, as in the unbounded fluid, mode-coupling leads now to the same expressions obtained in this section if $\nu$ is substituted by $\nu$ $+D$, where $D$ is the diffusion coefficient of the colloidal particle.

\section{RESULTS}

We have performed computer simulations to study the VACF and AVACF of a spherical particle suspended in a fluid, in contact with both a stick and a slip interface. To this end, we have used the lattice-Boltzmann model to describe the fluid. The state of the fluid is specified by the average number of particles, $n(\mathbf{c}, \mathbf{r}, t)$, with velocity $\mathbf{c}$, at each lattice site $\mathbf{r}$ at time $t$. The time evolution of the distribution functions is determined by the discretized analog of the Boltzmann equation [15], in which the $n(\mathbf{c}, \mathbf{r}, t)$ evolves in discrete time in two steps: propagation and collision. Collisions are specified such that the time evolution of the hydrodynamic fields satisfies the linearized Navier-Stokes equations for an isothermal fluid [15]. In this method, the solid surface which defines the colloidal particle and the confining surface are treated on the same footing, by modifying appropriately the collision step of the nodes adjacent to the surfaces [15]. Stick boundary conditions are ensured by imposing bounce-back of the incoming $n(\mathbf{c}, \mathbf{r}, t)$ along the links joining nodes of the surface and the fluid, while slip boundary conditions are enforced by specular reflection. Stick boundary conditions are always satisfied at the surface of the colloidal particle, while we change from stick to slip boundary conditions for the surface that bounds the fluid. The equations of motion of the particle are integrated using the self-consistent method described in Ref. [7]. We calculated the VACF by giving an initial velocity, $\vec{v}(0)$, to a colloidal particle in an otherwise quiescent fluid. In this description of the fluid, fluctuations are absent. Nonetheless, correlating the initial velocity of the freely moving particle $\vec{v}(0)$ with its subsequent values $\vec{v}(t)$ while it relaxes is, according to Onsager's regression hypothesis, equivalent to calculating the VACF in a "real" fluctuating fluid [16]. The initial velocity given to the particle is sufficiently small to ensure that its displacement is negligible compared to the lattice spacing. Our units are such that the mass of the lattice particles, the lattice spacing, and the time step are all unity, the density $\rho$ has a value of 24 , and the speed of sound $c$ has a value of $1 / \sqrt{ } 2$. The mass of the colloidal particle is chosen to correspond to neutral buoyancy. In all cases, the VACF is only calculated for times less than the time it takes for a sound wave to cross the system, so there are no finite-size effects to consider. In most of the cases, the asymptotic algebraic decay is reached within the simulation time, although in a few cases it is only the approach to it that is observable. We will start by analyzing the decay of the correlation functions of a disk in two dimensions, because a more detailed analysis is feasible since we can reach longer times than in their 3D counterparts. Afterwards, we will focus on the more realistic situation of a sphere in three dimensions, where we can compare with exact theoretical predictions [8].

Figures 3 and 4 show a log-log plot of the VACF for a disk in a 2D fluid both for slip and stick boundary conditions. For the sake of comparison, we also plot the VACF of the same particle in an unbounded fluid. The analysis of the preceding section assumed that only diffusion of velocity is relevant. This is indeed true in the asymptotic long-time regime, but at sufficiently short times, compressibility effects, related to the emission and reflection of sound waves, will modify the relaxation of the colloid. In Figs. 3 and 4, the diffusive decay of the VACF is only recovered at times of order $\tau_{w}$, when momentum has diffused the distance between the particle and the wall. This is also true for the particle in the unbounded medium, although in this case mo- 


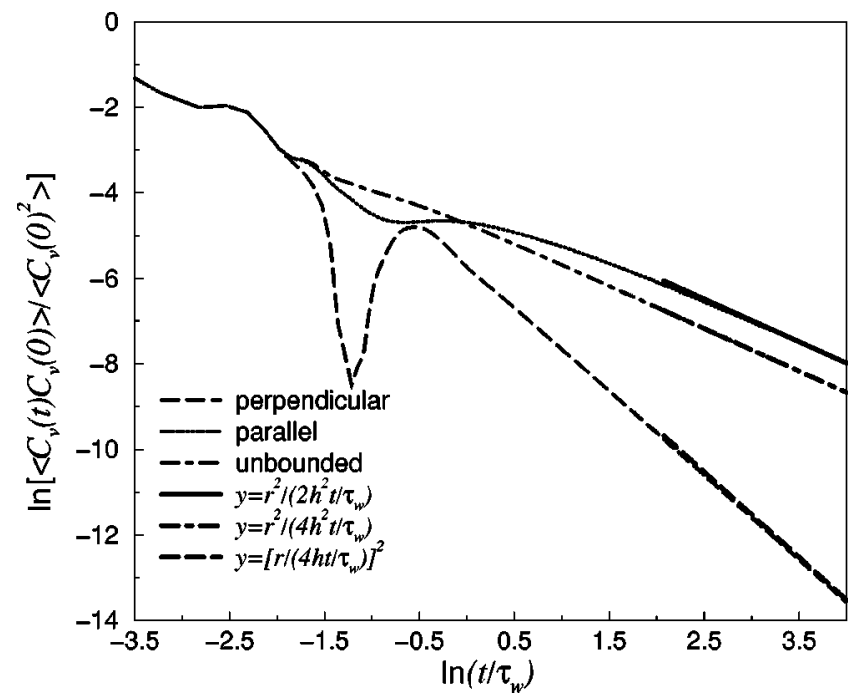

FIG. 3. Log-log plot of the VACF of a disk of radius $r=1.5$ at a distance $h=5.5$ from a slip surface, in a fluid of viscosity $\nu$ $=0.6$. The lines correspond to the theoretical prediction for the asymptotic decay.

mentum has to diffuse over the particle diameter, defining a characteristic diffusive time $\tau_{\infty} \equiv r^{2} / \nu$. Once the relaxation of the VACF is controlled by the diffusion of vorticity, algebraic decay is obtained, with powers given by Eqs. (5)(8). This suggests that the intuitive approach described in the preceding section is able to predict the decay of the different VACF's. For slip boundary conditions, we can also compare the amplitudes of the long-time tails. By fitting the algebraic decay, for a particle moving parallel to the interface we get an amplitude $A_{\|}^{\mathrm{sim}, \mathrm{sl}}=0.939$, while the theoretical expression Eq. (5) predicts $A_{\|}^{\text {th,sl }}=0.938$. For a colloid moving perpendicular to the interface, we should take into account that the actual distance between the colloidal particle and the wall is defined up to the resolution of the lattice itself [15]. This indeterminacy is important in this case because the distance to the wall is small, and a power of this distance enters in the

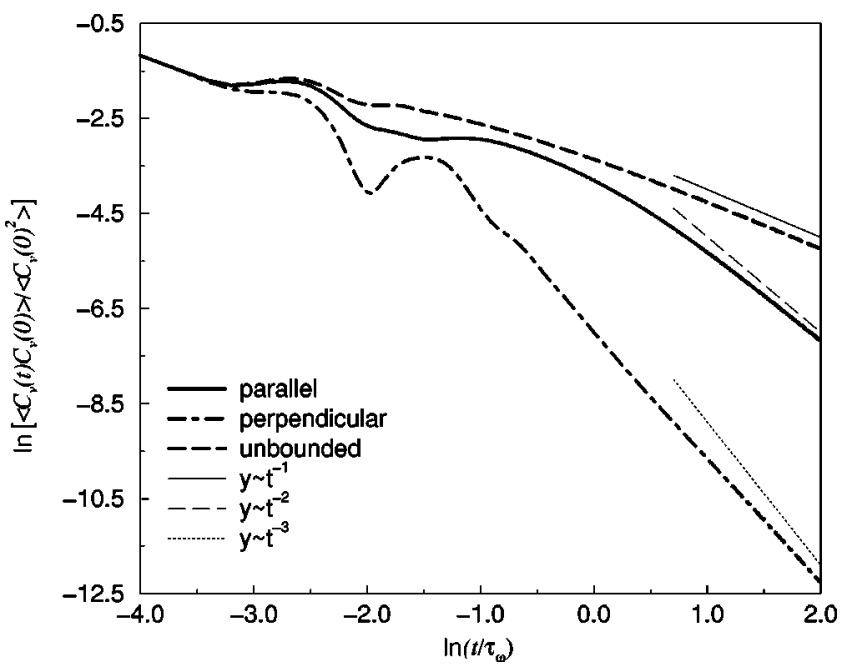

FIG. 4. Log-log plot of the VACF of a disk of radius $r=2.5$ at a distance $h=5.5$ from a stick surface, in a fluid of viscosity $\nu$ $=1 / 6$. The lines corresponding to the asymptotic decays are displaced to facilitate the comparison.

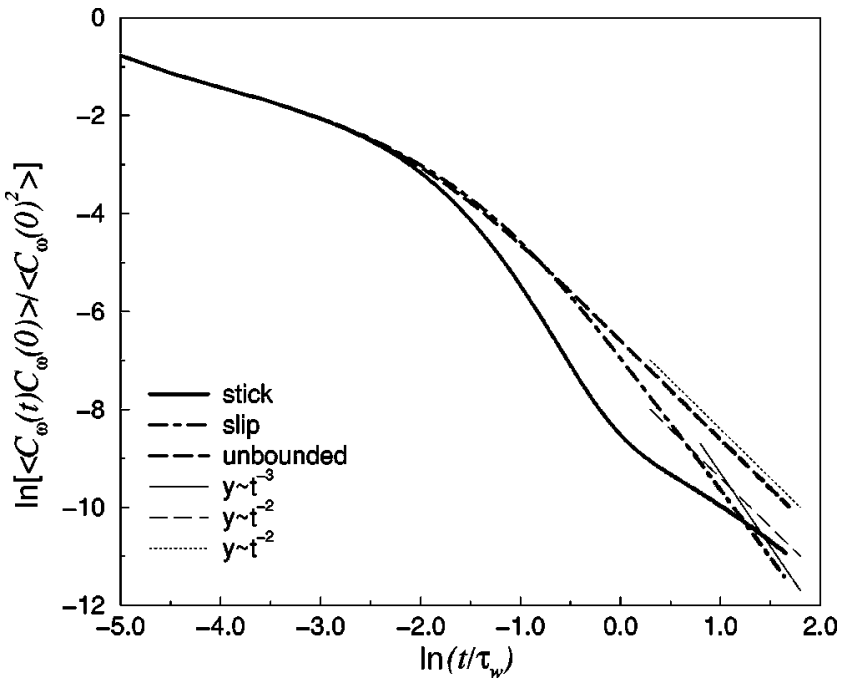

FIG. 5. Log-log plot of the AVACF of a disk of radius $r=2.5$ at a distance $h=5$ from the interface, in a fluid of viscosity $\nu=1 / 6$. The lines corresponding to the asymptotic decays are displaced to facilitate the comparison.

expressions for the amplitudes. In Fig. 3, the nominal distance between the disk and the interface is $h=5.5$. The simulation gives an amplitude $A_{\perp}^{\mathrm{sim}, \mathrm{sl}}=9.85$, while Eq. (6) predicts $A_{\perp}^{\text {th,sl }}=10.16$ for a real distance $h=5.1$. Note that the deviation with respect to the nominal distance is within half-lattice spacing. If we move away from the surface, this indeterminacy becomes less relevant. For example, for a nominal distance $h=13.5, A_{\perp}^{\mathrm{sim}, \mathrm{sl}}=69.0$, while $A_{\perp}^{\mathrm{th}, \mathrm{sl}}=71.2$. This shows that the deviations of a few percent that we get with respect to the theoretical predictions can be attributed to the discreteness of the lattice on which we simulate the fluid.

We can also give initially a certain angular velocity to an otherwise quiescent colloidal particle and inspect its decay. In Fig. 5 we show the log-log plot of the AVACF for both kinds of boundary conditions. The short-time decay is not purely diffusive due to the presence of the interface, but it does not lead to large distortions at short times. Surprisingly, it clearly shows that a slip surface induces a faster decay of the angular velocity than a stick interface, in agreement with the predictions of the preceding section, Eqs. (10) and (12).

We now turn to the more realistic case in which a spherical colloid of radius $a=0.5$ is confined by a planar interface. In Fig. 6, we show the log-log plot of the VACF's when the surface satisfies stick boundary conditions. The figure plots the absolute value of the VACF, because the velocity reverses its direction temporarily at short times. This is due to the small size of the particle and to the viscosity, which makes the particle more sensitive to the sound reflections from the wall at short times. The asymptotic long-time decay is algebraic and positive, and the exponents are again in agreement with Eqs. (7) and (8) of the preceding section, and with the predictions of Gotoh and Kaneda, Eq. (1). In this case, we can also compare with the theoretical predictions of Eq. (2). Again, we should take into account the uncertainty in the distance to the surface due to the discreteness of the lattice. The simulations reported in Fig. 6 correspond to the decay of a particle placed at a distance $h=2.5$ from the wall. However, in order to compare with the theoretical predic- 


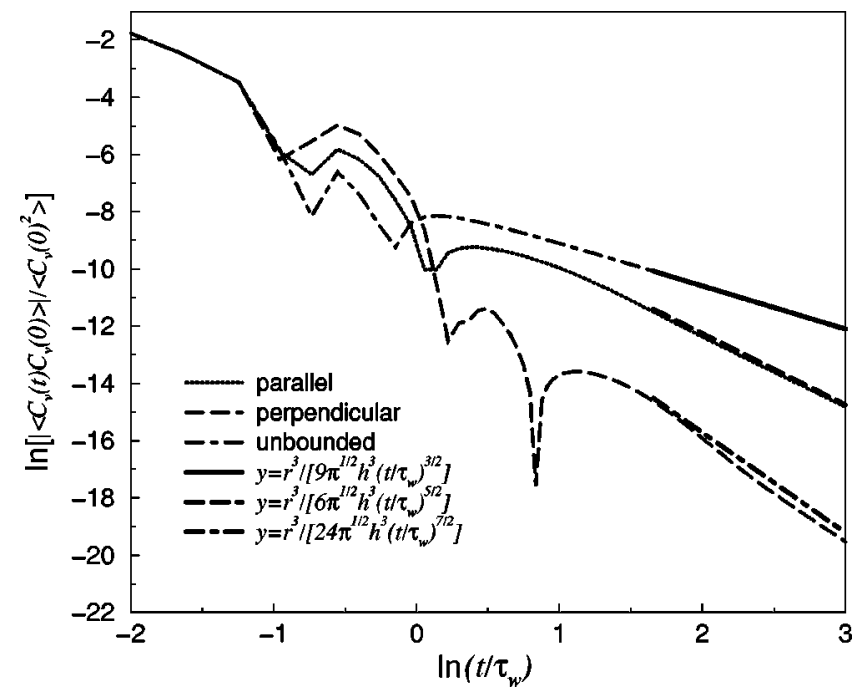

FIG. 6. Log-log plot of the VACF of a sphere of radius $r$ $=0.5$ at a distance $h=2.5$ from a solid interface, in a fluid of vis$\operatorname{cosity} \nu=0.6$. The straight lines correspond to the asymptotic, longtime behavior predicted by Gotoh and Kaneda.

tions for the amplitudes, we get the best agreement for a separation $h=2.42$, which is quite close to the nominal distance. By fitting the amplitudes of the asymptotic decay of the VACF's in Fig. 6, we get $A_{\|}^{\text {sim,st }}=0.253$ and $A_{\perp}^{\text {sim,st }}$ $=0.583$, while the theoretical expressions of Eq. (2) predict $A_{\|=}^{\text {st }}=0.2469$ and $A_{\perp}^{\text {st }}=0.6024$. Both simulation results agree with their theoretical counterparts to within $4 \%$. If we reduce the distance to $h=1.5$, we find agreement with the theoretical estimates for an effective separation of $h=1.4$, but with an error of 7\%. In Fig. 6, the asymptotes drawn correspond to the theoretical predictions of Gotoh and Kaneda. Although in the figure the asymptote corresponding to the perpendicular motion does not seem to agree well, this is due to the fact that it takes longer to reach the algebraic regime. To the best of our knowledge, this is the first direct test of the predictions of Ref. [8].

We have displayed in Fig. 7 the AVACF for a sphere of radius $a=0.5$. As opposed to the two-dimensional case, now the particle can rotate with its axis either parallel or perpendicular to the interface. For both kinds of boundaries, the powers obtained are again in agreement with the predictions of the preceding section, Eqs. (10)-(13). Contrary to the intuition, one can clearly see how the AVACF for a particle rotating with its axis parallel to a stick surface approaches the same algebraic decay as the one corresponding to an unbounded colloid, while the one corresponding to a slip interface decays faster. For the particular case in which the particle rotates with its axis perpendicular to a solid wall, we can also compare the theoretical prediction for the amplitude with the simulation results, because in this case the relative rotation of the source and image ensures that all the components of the velocity cancel at the surface. The nominal distance in this case is $h=2.5$, and we get the best agreement for $h=2.46$. In this case, the amplitude $A_{\perp}^{\text {sim,st }}=2.58$, while the theoretical prediction gives $A_{\perp}^{\text {th,st }}=2.56$. If we would have used $h=2.42$ as for the VACF, then $A_{\perp}^{\text {th,st }}=2.5$, which agrees with the simulation result within $3 \%$, consistent with the prediction for the VACF. In Fig. 7 one can see that the

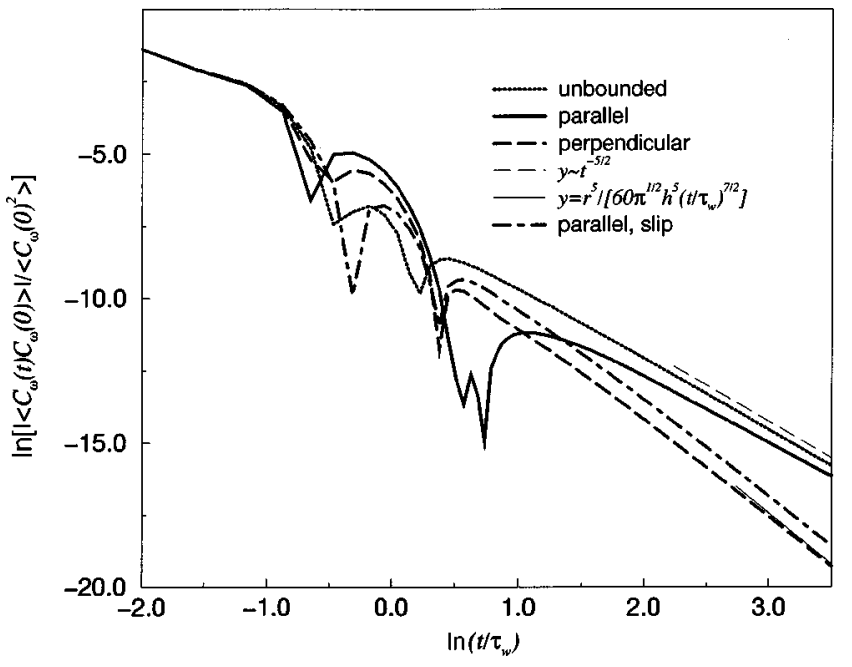

FIG. 7. Log-log plot of the AVACF of a sphere of radius $r$ $=0.5$ at a distance $h=2.5$ from the interface, in a fluid of viscosity $\nu=0.6$. All curves correspond to stick boundary conditions, except for the one in which it is explicitly indicated. The line $y \sim t^{-5 / 2}$ is displaced to facilitate the comparison.

AVACF for rotation perpendicular to the stick surface approaches the theoretical prediction, Eq. (13), asymptotically.

Finally, the presence of an interface will induce rotation in an initially translating disk parallel to the wall, and translation in an initially rotating disk. The decay of these cross motions for a 2D disk is shown in Fig. 8 for both kinds of boundary conditions. Initially, the corresponding velocity is zero, which explains the short-time increase in the plots. The decay at long times is also algebraic, with the same exponent, irrespective of the particular boundary condition considered. In fact, the angular velocity in Fig. 8(a) is determined by the vorticity induced by the image source. On the other hand, the velocity observed in Fig. 8(b) is due to the flow field generated by the image dipole. In both cases, it decays as $t^{-2}$ in 2D, in general as $t^{-d / 2+1}$, i.e., the algebraic decay due to a dipolar source. Note that this is independent of the specific boundary conditions, and it arises purely as a consequence of the confinement. However, for stick boundary conditions the induced velocity reverses its direction of motion at long times due to the influence of the sound reflected back at the boundary. Correspondingly, in Fig. 8 we have displayed the absolute value of the induced velocity for a stick interface.

\section{DISCUSSION}

In this paper we have studied the effect of an interface on the short-time dynamics of a suspended particle. The rotational and translational diffusion become anisotropic due to the presence of a surface, which induces, in general, a faster decay of motions perpendicular to it.

We have also shown that the asymptotic algebraic decay of the velocity of a particle is sensitive to the specific boundary conditions considered. In most cases, stick boundary conditions induce a faster decay of the hydrodynamic excitations than slip boundary conditions, and the AVACF decays with one power faster than the corresponding VACF. However, we also find that for rotation with the axis parallel 

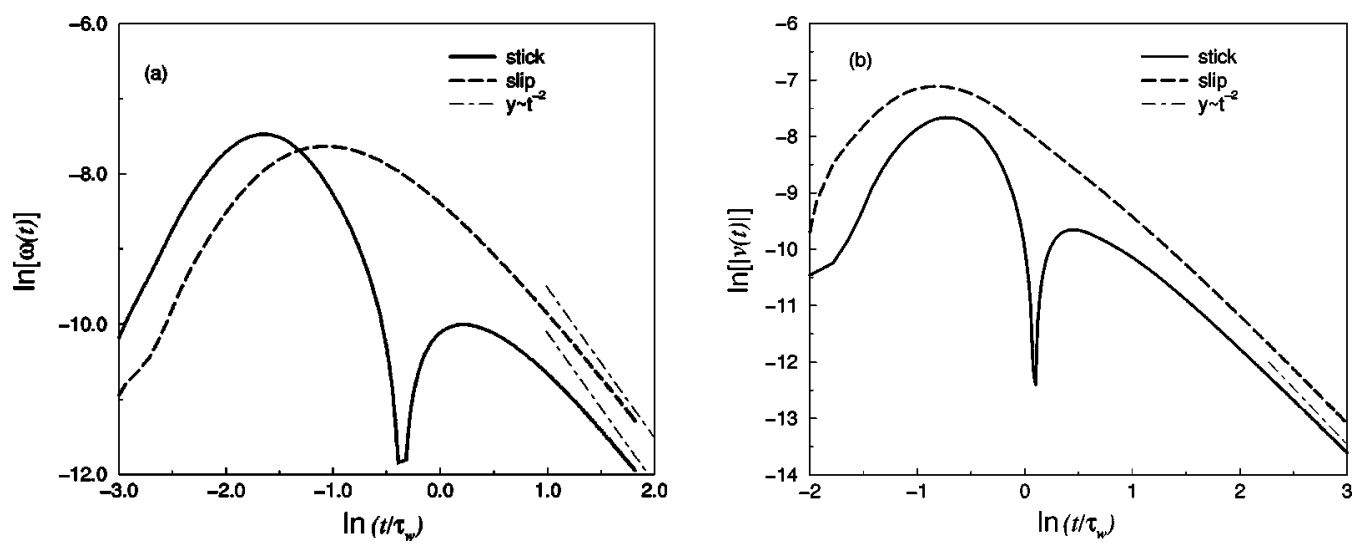

FIG. 8. Log-log plot of the induced cross motion of a disk of radius $r=2.5$ at a distance $h=4.5$ from the interface, in a fluid of viscosity $\nu=1 / 6$. For stick boundary conditions we plot the absolute values, because the induced velocities reverse their direction at long times. Velocities and angular velocities are expressed in the units introduced in Sec. III. (a) Angular velocity of an initially translating disk. (b) Velocity of an initially rotating disk. The lines corresponding to the asymptotic decays are displaced to facilitate the comparison.

to the interface the behavior is unexpected: for a stick surface the AVACF decays slower than for slip boundary conditions.

The decay of the perturbations is dominated by momentum diffusion at long times. For this reason, the onset of the asymptotic regime only occurs for times of order $\tau_{w}$. From the expressions for the amplitudes in Sec. II, it would seem that the larger $h$ is, the larger is the amplitude of the algebraic tail. However, since it is necessary to wait longer to enter the asymptotic regime, the actual amplitude is smaller. As can be seen in Table II, if we express the velocity correlation functions in units of $t / \tau_{w}$, the amplitude always decreases with $h$ (as $h^{-d}$ for rotation and $h^{-(d+2)}$ for rotation). Of course, the velocity and angular momentum of a particle in an unbounded fluid would exhibit the usual algebraic decay for times larger than $\tau_{\infty}$. This implies that for distances $h \gg r$, we will first observe the usual $t^{-d / 2}$ decay and, only after a time that is a factor $(h / r)^{2}$ larger, a crossover to the behavior induced by the presence of the interface.

We focused our analysis on the single-particle case. However, our results should also apply to the case of a colloidal suspension close to an interface. Due to the linearity of hydrodynamics, the power law of the VACF will not be modified. Only its amplitude will differ from the prediction of Gotoh and Kaneda [8]. If the particles can move in random directions, at long times the lowest decaying perturbation will be the dominant one. As an example, for the case of translation in $3 \mathrm{D}$, the $t^{-5 / 2}$ decay related to the motion parallel to the surface will be dominant at long times. In order to extract the decay of the perpendicular motion, it will be necessary to restrict the motion of the particles, e.g., by applying external fields.

From the experimental point of view, it may be easier to analyze the mean-square displacement, $\Delta \equiv\langle[\vec{r}(t)$

TABLE II. Scaling of the amplitudes of the VACF with $h$ at the onset of the asymptotic regime, when time is expressed in units of the diffusive time $\tau_{w}$. For $d=2, C_{\omega}^{\perp}$ is not defined.

\begin{tabular}{lllll}
\hline \hline & $C_{v}^{\|}$ & $C_{v}^{\perp}$ & $C_{\omega}^{\|}$ & $C_{\omega}^{\perp}$ \\
\hline Slip & $h^{-d}$ & $h^{-d}$ & $h^{-(d+2)}$ & $h^{-(d+2)}$ \\
Stick & $h^{-d}$ & $h^{-d}$ & $h^{-(d+2)}$ & $h^{-(d+2)}$ \\
\hline \hline
\end{tabular}

$\left.-\vec{r}(0)]^{2}\right\rangle$, which is related to the integral of the VACF,

$$
\Delta(t)=2 d t\left(\int_{0}^{t} C_{v}\left(t^{\prime}\right) d t^{\prime}-\frac{1}{t} \int_{0}^{t} t^{\prime} C_{v}\left(t^{\prime}\right) d t^{\prime}\right) .
$$

In Fig. 9 we show the time-dependent diffusion coefficient, $D(t) \equiv d(\Delta(t)) / d t$, for the motion of a single sphere both in an unbounded fluid and in the presence of a single wall. We have considered separately the case of parallel and perpendicular motion with respect to the interface. The presence of the wall does not introduce major qualitative features, except for the saturation of the diffusion coefficient at a smaller value and for the more pronounced peak at short times. Therefore, it is the analysis of the time derivatives of $\Delta(t)$ which will show more clearly the qualitative differences induced by the wall on the dynamics of the particles. At finite volume fractions the asymptotic value will be modified, but the qualitative features will remain.

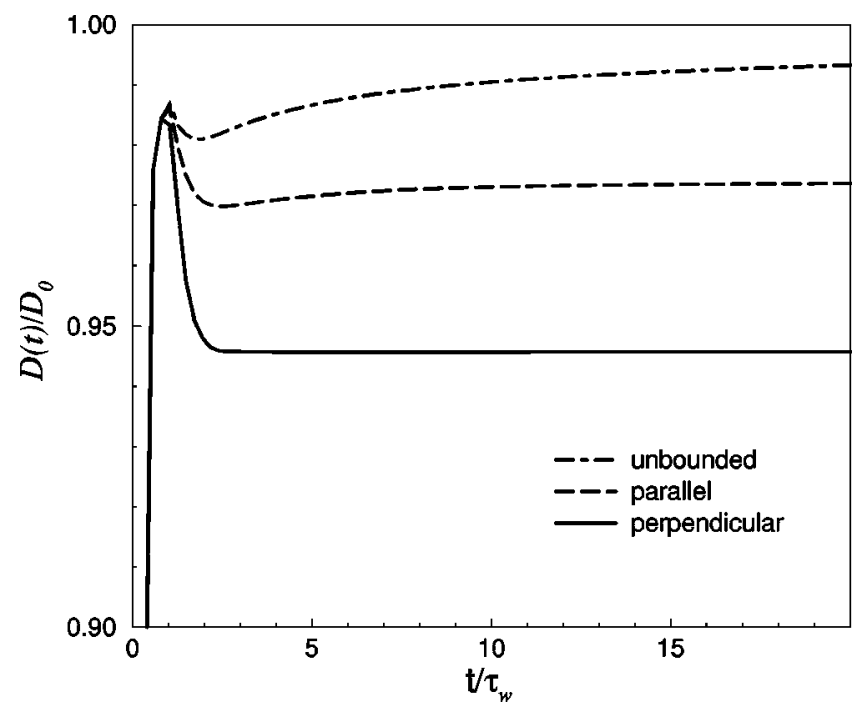

FIG. 9. Mean-square displacement of a spherical particle of radius $r=0.5$ at a distance $h=2.5$ from a stick surface, in a fluid of viscosity $\nu=0.6$. 
In this paper we have considered the situation in which a single surface is present. We have also presented an intuitive picture along with the simulations, which relies on the method of images. One might be tempted to apply the same method for a colloidal suspension confined between two walls, or inside a cylinder. However, in this case there may be situations where sound modes propagate diffusively if stick boundary conditions apply [9]. Then, the assumption that the dynamics is controlled by vorticity is no longer valid and the algebraic decays for the VACF will differ from the predictions based on the image scheme obtained here.

\section{ACKNOWLEDGMENTS}

We thank J. Polson for a careful reading of the manuscript. The work of the FOM Institute is part of the scientific program of FOM and is supported by the Nederlandse Organisatie voor Wetenschappelijk Onderzoek (NWO). Computer time on the CRAY-C98/4256 at SARA was made available by the Stichting Nationale Computer Faciliteiten (Foundation for National Computing Facilities). I.P. acknowledges E.U. for financial support (Contract No. ERBFMBICT-950433), and the FOM Institute for its hospitality.
[1] W. B. Russel, D. A. Saville, and W. R. Schowalter, Colloidal Dispersions (Cambridge University Press, Melbourne, 1989); J. K. G. Dhont, An Introduction to the Dynamics of Colloids (Elsevier, Amsterdam, 1996).

[2] J. X. Zhu, D. J. Durian, J. Müller, D. A. Weitz, and D. J. Pine, Phys. Rev. Lett. 68, 2559 (1992).

[3] M. H. Kao, A. G. Yodh, and D. J. Pine, Phys. Rev. Lett. 70, 242 (1993).

[4] J. R. Blake, Proc. Cambridge Philos. Soc. 70, 303 (1971).

[5] B. J. Alder and T. E. Wainwright, Phys. Rev. A 1, 18 (1970).

[6] B. J. Berne, J. Chem. Phys. 56, 2164 (1972).

[7] C. P. Lowe, D. Frenkel, and A. J. Masters, J. Chem. Phys. 103, 1582 (1995).
[8] T. Gotoh and Y. Kaneda, J. Chem. Phys. 76, 3193 (1982).

[9] M. H. J. Hagen, I. Pagonabarraga, C. P. Lowe, and D. Frenkel, Phys. Rev. Lett. 78, 3785 (1997).

[10] M. H. Ernst, E. H. Hauge, J. M. J. van Leeuwen, Phys. Rev. Lett. 25, 1254 (1970).

[11] P. M. Morse and H. Feshbach, Methods of Theoretical Physics (McGraw-Hill, New York, 1953).

[12] C. Pozrikidis, Phys. Fluids A 1, 1508 (1989).

[13] A. Masters, J. Chem. Phys. 105, 9695 (1996).

[14] Y. Pomeau and P. Résibois, Phys. Rep. 19, 63 (1974).

[15] A. J. C. Ladd, J. Fluid Mech. 271, 285 (1994).

[16] L. Onsager, Phys. Rev. 37, 405 (1931). 